\section{P188 Thumbs Up for Healthy Choices: Making the Healthy Choice, the Easy Choice in Utah's Food Pantries}

Casey Coombs, RDN, casey.coombs@usu.edu, Utah SNAPEd, Utah State University, 8749 Old Main Hill, Logan, UT 84322; Mateja R. Savoie-Roskos, PhD, MPH, RDN, CNP, Utah State University; Heidi LeBlanc, MS, Utah SNAP-Ed, Utah State University

Background (Background, Rationale, Prior Research, and/or Theory): Food pantry users are often assumed to have poor diet quality. Identifying effective strategies that increase the selection of healthy foods by pantry users may help improve their dietary intake. Utah's Supplemental Nutrition Assistance Program-Education (SNAP-Ed) developed the Thumbs Up for Healthy Choices program which aims to make the healthy choice, the easy choice in pantries. Thumbs Up for Healthy Choices uses nudge strategies including shelf signs, recipe cards, and posters to increase the visibility of healthy options.

Objective: The objective of this study was to evaluate the effectiveness of the Thumbs Up for Healthy Choices program on food pantry users' selection and use of targeted foods.

Study Design, Setting, Participants, Intervention: A convenience sample of 457 food pantry users in six urban Utah food pantries were surveyed. Participants were asked about their familiarity with the Thumbs Up program, barriers to making healthy choices, selection of promoted items, use of foods and recipes at home, as well as the impact of the program on dietary intake.

Outcome Measures and Analysis: Frequencies and descriptive statistics were analyzed. Chi-square associations were used to identify relationships between demographic characteristics and program impact. Logistic regression was used to analyze the effect of exposure on program impacts.

Results: Eighty-four percent of respondents agree or strongly agree that Thumbs Up makes it easier to make healthy choices. Sixty-seven percent of respondents agree or strongly agree they eat healthier since Thumbs Up was implemented. Hispanics were more likely than nonHispanics to report healthier eating for themselves and their families $(P=.002, P=.001)$. After controlling for age, ethnicity, and gender, program exposure was significantly associated with using targeted foods at home $(P=<.001)$ and selecting foods with Thumbs Up signs $(P=.002)$.

Conclusions and Implications: These results suggest that the Thumbs Up for Healthy Choices program positively impacts food pantry users' selection of targeted healthy foods. Nudge programs, including Thumbs Up for Healthy Choices, are low-input strategies that may improve food pantry users' selection and consumption of healthy foods.

Funding: Supplemental Nutrition Assistance Program-Education.
P189 UC CalFresh Nutrition Education Program Empowering Low-Income Families with Knowledge and Skills About Food Resource Management

Deepa Srivastava,PhD, dsr@ucdavis.edu, University of California Cooperative Extension, 4437-B S Laspina Street, Tulare, CA 93274; Barbara Mknelly, MS

Objective: To improve food resource management behaviors essential to maximizing use of limited resources, supporting a healthy diet, and improving food security among low-income families.

Target Audience: Supplemental Nutrition Assistance Program Education (SNAP-Ed) eligible/ low-income adults/ families in Tulare County, California.

Theory, Prior Research, Rationale: The UC CalFresh NEP is guided by the SNAP-Ed Evaluation Framework.

Description: During the FFY2017, a four-week seriesbased Plan Shop Save Cook (PSSC) curriculum was administered to UC CalFresh program participants. The program included the following components: plan meals, use a shopping list, understand and use food labels, save money, and cook a meal.

Evaluation: A pre-post evaluation was conducted to determine behavioral change in planning meals, use of shopping list, use of food labels, saving money, and cooking a meal. Of the total participants $(n=98)$ who completed the pre-post survey, majority were Hispanic-Latino (94\%) and female (92\%). Paired sample t-test analysis indicated statistically significant results for the following behaviors: shop with a list (t $(97)=2.333, P=.022)$, think about health choices $(\mathrm{t}(97)=2.303, P=.023)$, plan meals $(\mathrm{t}$ $(97)=3.790, P=.000)$, use nutrition facts label $(\mathrm{t}$ $(97)=4.519, P=.000)$, use MyPlate $(\mathrm{t}(97)=6.764, P=.000)$, and all five behaviors ( $\mathrm{t}(97)=4.619, P=.000)$.

Conclusions and Implications: Through a series of nutrition classes, UC CalFresh NEP improved knowledge and skills of adult participants to plan tasty, cost-effective, and nutritious meals. Findings provide essential insight into behavioral strategies (e.g. shopping with a list, planning meals, reading nutrition facts labels) for communicating food resource management messages to low-income families. Nutrition education professionals can use these food resource management strategies to tailor educational messages for limited resources families.

Funding: California SNAP-Ed (UC CalFresh NEP).

\section{P190 Utilization of a Near-Peer Nutrition Education Program for Undergraduates}

Crystal C.Douglas,PhD, RDN, ccdouglas@shsu.edu, Sam Houston State University, 1700 University Avenue, Huntsville, TX 77341; Simone P. Camel, PhD, RDN

Objective: Literature supports the use of peer-mentors to provide education and guidance for improving health behaviors; however, there is a paucity of research specifically utilizing motivational interviewing and counseling 